\title{
Supersonic Jet Noise Reductions Predicted With Increased Jet Spreading Rate
}

Milo D. Dahl

Lewis Research Center

Cleveland, Ohio

and

Philip J. Morris

Pennsylvania State University

University Park, Pennsylvania

Prepared for the

Joint Fluids Engineering Conference

cosponsored by the American Society of Mechanical Engineers

and the Japan Society of Mechanical Engineers

Hilton Head, South Carolina, August 13-18, 1995
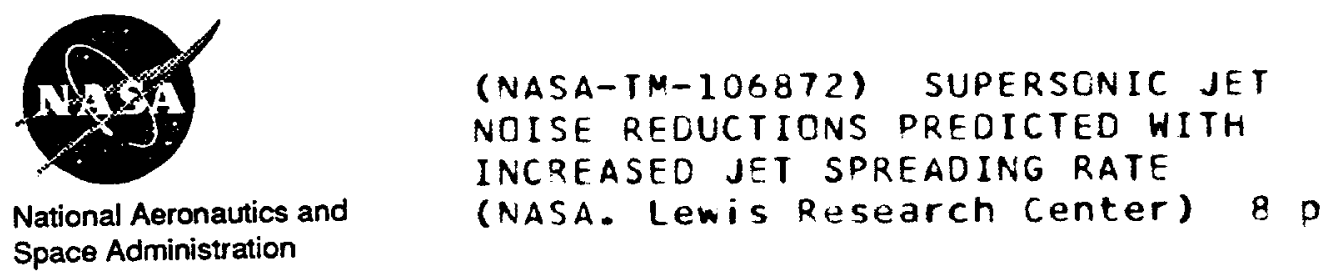

Unclas 


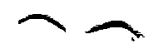




\title{
SUPERSONIC JET NOISE REDUCTIONS PREDICTED WITH INCREASED JET SPREADING RATE
}

\author{
Milo D. Dahl \\ NASA Lewis Research Center \\ Cleveland, $\mathrm{OH} 44135$ \\ Philip J. Morris \\ Penn State University \\ University Park, PA 16802
}

\begin{abstract}
In this paper, predictions are made of noise radiation from single, supersonic, axisymmetric jets. We examine the effects of changes in operating conditions and the effects of simulated enhanced mixing that would increase the spreading rate of the jet shear layer on radiated noise levels. The radiated noise in the downstream direction is dominated by mixing noise and it is well described by the instability wave noise radiation analysis. A numerical prediction scheme is used for the mean flow providing an efficient method to obtain the mean flow development for various operating conditions and to simulate the enhanced mixing. Using far field radiated noise measurements as a reference, the calculations predict that enhanced jet spreading results in a reduction of radiated noise.
\end{abstract}

\section{INTRODUCTION}

Recent interest in the development of a new High Speed Civil Transport has renewed research efforts to make the aircraft environmentally compatible. One issue is the level of noise generated by the supersonic jets exhausting from the propulsion systems. Supersonic jets are intense noise generators and means must be found to modify the noise generation process to reduce radiated noise levels in order for the aircraft to meet community noise regulations. A recent review by Seiner and Krejsa [1989] discusses the challenge of reducing supersonic jet noise associated with both mixing and shocks while maintaining acceptable propulsion system performance requirements. In this paper, we focus on the issue of mixing noise and the concept that the promotion of rapid mixing of the jet shear layer is an effective means of reducing radiated levels of mixing noise.

At present, the primary means of promoting enhanced jet shear layer spreading is either through acoustical or mechanical excitation. It is well known that subsonic jet mixing can easily be enhanced through acoustic excitation; but, for supersonic jets, acoustic excitation is much less effective (Lepicovsky et al. [1985]). Mechanical means for promoting jet shear layer spreading were found to be more effective in supersonic jets than acoustic excitation. For example, Ahuja and Brown [1989] inserted small tabs into the jet flow at the lip of the nozzle exit. Alternatively, Gutmark et al. [1989] used nozzle shaping, such as elliptic and rectangular nozzles, to promote increased shear layer spreading. Experiments to measure the noise from supersonic jets with mechanical tabs have been performed by Ahuja [1993] and Kobayashi et al. [1993]. In both studies, the jets contained shocks and for the most part the overall noise reduction was primarily due to the reduction of noise associated with shocks. However, the studies did show decreases in noise levels in the downstream direction. Measurements of noise from perfectly expanded jets from elliptic nozzles (Seiner and Ponton [1991]) showed noise reductions compared to the equivalent axisymmetric jet. In this case, the results depended on the azimuthal angle.

While there have been noise predictions for both supersonic axisymmetric and elliptic jets, noise predictions have not been made for supersonic jets with enhanced mixing. It is the purpose of this paper to make noise predictions for single, supersonic, axisymmetric jets for given operating conditions and evaluate the resulting noise reduction from simulated enhanced mixing that increases the spreading rate of the jet shear layer. 
It is known that instability waves are the dominant source of mixing noise radiating into the downstream arc of a supersonic jet when the phase velocities of the wave are supersonic relative to ambient conditions. The analysis to predict the noise radiated to the far field is based on the determination of the axial growth and decay of the instability wave in the jet shear layer. In order to complete the analysis, the mean flow properties are needed. In the next section, we describe the numerical prediction method for the mean flow development. This provides an efficient method to obtain the mean flow for various operating conditions and to simulate shear layer growth due to enhanced mixing. The procedures to calculate the evolution of instability waves and their radiated noise are presented next. This is followed by a comparison of calculated far field radiated noise patterns to measured supersonic jet data in order to calibrate one unknown constant. Based on those results, we then compare calculated results for a jet with simulated enhanced mixing to measured data. Finally, we present a numerical study of the changes in radiated noise levels with changes in jet spreading rate.

\section{MEAN FLOW PREDICTIONS}

This section discusses the numerical procedure used to calculate the mean flow development of a compressible, axisymmetric jet. The jet flow is assumed to be perfectly expanded, thus the jet static pressure is matched to the ambient pressure. Often in the analysis of the mixing noise generation in single supersonic axisymmetric jets, the axial variation in the mean velocity profile is based on simple analytic functions with axially varying parameters described by equations fitted to measured data. In supersonic jets where temperature profiles are not easily measured, Crocco's relation is used to obtain mean density profiles. However, in order to predict radiated jet noise for a variety of operating conditions, it is appropriate to use numerically generated mean flow profiles. Thus, we use a set of compressible, Reynolds averaged, boundary layer equations with a modified mixing length model to determine the Reynolds stresses as the basis for the numerical mean flow analysis. To simulate the effects of enhanced mixing, the coefficient of the mixing length model is increased to provide a larger eddy viscosity which causes the jet shear layer to spread faster.

\subsection{Turbulence Model}

The development of a prediction scheme for the mean flow properties of a jet was completed with the capability to compute both single stream axisymmetric jets and dual stream coaxial jets, Dahl [1994]. Simplicity and robustness were emphasized in order to calculate the mean velocity and density and their derivatives accurately for later usage in the instability wave calculations. This led us to choose a simple turbulence model, resulting in a high level of empiricism.

The compressible equations of motion are simplified to the boundary layer form. The assumption is also made that the density-velocity correlations may be neglected. For the single jet case, the Reynolds stress and the heat flux terms are described by the following mixing-length model:

$$
-\rho \overline{u^{\prime} v^{\prime}}=\mu_{\mathrm{T}} \frac{\partial u}{\partial r}
$$

and

$$
-\rho c_{p} \overline{v^{\prime} T^{\prime}}=\frac{c_{p} \mu_{\mathrm{T}}}{\operatorname{PrT}_{\mathrm{T}}} \frac{\partial T}{\partial r}
$$

with

$$
\mu_{\mathrm{T}}=\rho\left(\kappa C_{1} C_{2} \ell\right)^{2}\left|\frac{\partial u}{\partial r}\right| \text {. }
$$

$\ell$ is a characteristic mixing length scale given by

$$
\ell=\frac{\Delta U}{|\partial u / \partial r|_{\max }}
$$

and $\kappa$ is a constant to simulate the effects of enhanced mixing.

$$
\begin{array}{ll}
\kappa=1, & \text { no enhanced mixing } \\
\kappa>1, & \text { enhanced mixing }
\end{array}
$$

The factor $C_{1}$ is the incompressible part of the mixing length constant. It depends on the the velocity ratio $U_{\infty} / U_{j}$ and the density ratio $\rho_{\infty} / \rho_{j}$. The $C_{2}$ factor is the compressible part of the mixing length constant. Its purpose is to decrease the growth of the shear layer as compressibility effects become important. It depends on a Mach number in a frame of reference convecting with the real phase speed of a growing disturbance in the shear layer. This convected Mach number depends on the velocity ratio, the density ratio, and the Mach number of the jet. Thus, both factors depend on the flow conditions and a calibration procedure was developed to obtain empirical equations to describe both factors. The details are given in Dahl [1994].

\subsection{Numerical Method}

The numerical method initially follows the stream function approach given by Crawford and Kays [1976]. The equations of motion in boundary layer form are transformed into stream function coordinates using

$$
r \rho u=\frac{\partial \Psi}{\partial r} \quad \text { and } \quad r \rho \tilde{v}=-\frac{\partial \Psi}{\partial x}
$$

where $\tilde{v}$ is the mass averaged radial component of the mean velocity. The boundary layer equations become

$$
\begin{gathered}
\frac{\partial u}{\partial x}=\frac{\partial}{\partial \Psi}\left[r^{2} \rho u \mu_{\mathrm{eff}} \frac{\partial u}{\partial \Psi}\right] \\
\frac{\partial H}{\partial x}=\frac{\partial}{\partial \Psi}\left[r^{2} \rho u \frac{\mu_{\mathrm{eff}}}{\operatorname{Pr}_{\mathrm{eff}}} \frac{\partial H}{\partial \Psi}\right] \\
+\frac{\partial}{\partial \Psi}\left[r^{2} \rho u^{2}\left(\mu_{\mathrm{eff}}-\frac{\mu_{\mathrm{eff}}}{\mathrm{Pr}_{\mathrm{eff}}}\right) \frac{\partial u}{\partial \Psi}\right]
\end{gathered}
$$


where $H$ is the total mean enthalpy and

$$
\mu_{\text {eff }}=\mu+\mu_{\mathrm{T}}
$$

and

$$
\operatorname{Pr}_{\text {eft }}=\frac{1+\frac{\mu_{\mathrm{T}}}{\mu}}{\frac{1}{\operatorname{Pr}}+\frac{1}{\operatorname{Pr}_{\mathrm{T}}} \frac{\mu_{\mathrm{T}}}{\mu}}
$$

The numerical equations are derived by using fully implicit differencing on equations (7) and (8). This insures that the numerical problem is inherently stable. Since both the mean flow and instability wave problems are calculated using the same grid, maintaining sufficient grid resolution required that the problems be solved on a fine, evenly spaced r-grid. Details of the finite difference algorithm and solution procedure are given in Dahl [1994] as are favorable comparisons between calculated and measured data for various subsonic and supersonic jets.

\section{INSTABILITY WAVES AND RADIATED NOISE}

It is well known that thin free shear layers containing an inflection point in the mean velocity profile are inherently unstable even in the absence of viscosity. An instability wave in the shear layer initially grows rapidly. As the shear layer grows, the wave growth rate decreases. Eventually, the shear layer is too thick to support unstable waves and the wave amplitude decreases until it disappears. This instability wave process is assumed to be governed by the linearized, inviscid, compressible equations of motion.

For slowly diverging jet flows, two solutions are created that apply to separate but overlapping regions. Following the approach of Tam and Burton [1984], the inner region, including the jet flow and the immediate region just outside the jet, has different length scales between the radial and the axial directions that leads to a multiple scales expansion of the governing equations. With the pressure disturbances represented as

$$
\begin{aligned}
p^{\prime}(r, \theta, x, t) & =\sum_{m=0}^{\infty} \delta_{m}(\varepsilon) p_{m}(r, s) \\
& \times \exp \left[i\left(\frac{\phi(s)}{\varepsilon}+n \theta-\omega t\right)\right],
\end{aligned}
$$

the lowest order set of equations may be reduced to

$$
\begin{aligned}
\frac{\partial^{2} p_{0}}{\partial r^{2}} & +\left[\frac{1}{r}+\frac{2 \alpha}{\omega-\alpha \bar{u}} \frac{\partial \bar{u}}{\partial r}-\frac{1}{\bar{\rho}} \frac{\partial \bar{\rho}}{\partial r}\right] \frac{\partial p_{0}}{\partial r} \\
& +\left[\bar{\rho} M_{j}^{2}(\omega-\alpha \bar{u})^{2}-\frac{n^{2}}{r^{2}}-\alpha^{2}\right] p_{0}=0
\end{aligned}
$$

which is known as the compressible Rayleigh Equation. In equation (11), $\delta_{m}(\varepsilon)$ are the gauge functions of the aymptotic expansion in the small parameter $\varepsilon$ where $\delta_{0}(\varepsilon)=1, s$ is the slow variable to recognize the slow mean flow development in the axial direction $s=\varepsilon x, \phi(s)$ is an axial phase function related to the axial wavenumber $\alpha$ by $d \phi / d s=\alpha(s), n$ is the azimuthal mode number, and $\omega$ is the radian frequency.

The general solution to equation (12) is written

$$
p_{0}(r, s)=A_{0}(s) \zeta_{1}^{p}(r, s)+B_{0}(s) \zeta_{2}^{p}(r, s) .
$$

As $r \rightarrow \infty$, the flow outside the jet becomes uniform and the solution to equation (12) may be written in the form

$$
p_{0}=A_{0} H_{n}^{(1)}(i \lambda(\alpha) r)+B_{0} H_{n}^{(2)}(i \lambda(\alpha) r)
$$

where

$$
\lambda(\alpha)=\left[\alpha^{2}-\bar{\rho}_{\infty} M_{j}^{2}\left(\omega-\alpha \bar{u}_{\infty}\right)^{2}\right]^{1 / 2}
$$

and $H_{n}^{(1)}$ and $H_{n}^{(2)}$ are $n$ th-order Hankel functions of the first and second kind, respectively. Thus, for large $r$, equation (13) must tend to equation (14). As $r \rightarrow 0$, equation (13) must be finite.

In the outer region, which slightly overlaps the inner region, the governing equations control disturbances that are acoustic in nature. These disturbances have the same length scales in all directions; hence, all coordinates are treated equally. To create an outer solution in a form that allows it to be asymptotically matched to the inner solution, we use the axial coordinate $s=\varepsilon x$ and the radial coordinate $\bar{r}=\varepsilon r$. The solution is obtained by Fourier transforming the outer region governing equations in the $s$ direction. After considerable algebra, the lowest order outer solution is

$$
p(r, \theta, x, t)=\int_{-\infty}^{\infty} g(\eta) H_{n}^{(1)}(i \lambda(\eta) r) e^{i \eta x} e^{i n \theta} e^{-i \omega t} d \eta
$$

where

$$
g(\eta)=\frac{1}{2 \pi} \int_{-\infty}^{\infty} \tilde{A}_{0}(\varepsilon x) e^{i \phi(\varepsilon x) / \varepsilon} e^{-i \eta x} d x
$$

The two solutions are matched asymptotically in an overlap region. To lowest order, we find that

$$
A_{0}(s)=\tilde{A}_{0}(s) \quad \text { and } \quad B_{0}(s)=0 .
$$

With the condition of finite value at $r=0$, equation (12) has become an eigenvalue problem with solutions only for certain values of the eigenvalue $\alpha$. We have used a finite-difference approximation to discretize the problem. The eigenvalue is found from the resulting diagonal matrix using a Newton-Raphson iteration for refinement.

Since the rate of spread of the jet is slow for high speed jets and $\varepsilon$ is very small, $\tilde{A}_{0}(\varepsilon x)$ in equation (17) is taken to be constant. Furthermore with $\alpha(x)$ found from the solution of equation (12) at every axial location, the 
axial phase function is found from $\phi(\varepsilon x) / \varepsilon=\int_{0}^{x} \alpha(x) d x$. We can then solve for $g(\eta)$ in equation (17), the Fourier transform of the instability wave, and subsequently the near field pressure disturbance is found from equation (16). To obtain the pressure in the far field, equation (16), in spherical coordinates, is approximated by the method of stationary phase. The resulting sound power radiated per unit solid angle is

$$
D(\psi)=\frac{1}{2}|p|^{2} R^{2}=2 \frac{|g(\bar{\eta})|^{2}}{\left[1-M_{\infty}^{2} \sin ^{2} \psi\right]} .
$$

The stationary point is given by

$$
\bar{\eta}=\frac{\bar{\rho}_{\infty}^{1 / 2} M_{j} \omega \cos \psi}{\left(1-M_{\infty}^{2}\right)\left(1-M_{\infty}^{2} \sin ^{2} \psi\right)^{1 / 2}}-\frac{\bar{\rho}_{\infty} M_{j}^{2} \bar{u}_{\infty} \omega}{1-M_{\infty}^{2}}
$$

and $\psi$ is the polar angle.

To validate the mean flow and noise radiation prediction schemes, Dahl [1994] compared calculated results using numerically generated mean flow profiles to measured data (Seiner and Ponton [1985]) and to calculated results using analytically generated mean flow profiles (Seiner et al. [1993]) and found favorable comparisons in the far field radiated noise patterns even though the numerically generated mean flows had longer potential core lengths than the measured data. However, the calculated initial spreading of the jet was similar to measured data and encompassed the region of maximum growth of the instability wave. Thus, we assume that given the jet operating conditions, we can calculate the initial jet spreading with sufficient accuracy to obtain reasonable results from the instability wave noise radiation analysis.

\section{PREDICTED AND MEASURED SOUND}

\subsection{Variations in Operating Conditions}

Figure 1 shows a comparison between calculated and measured far field noise radiation patterns for three $M_{j}=2$, hot jet cases given in Seiner et al. [1992]. The calculated results are for the first helical mode instability wave at Strouhal number 0.11 . The calculations are normalized to the measured peak level. By doing this, it is found that the constant in the problem, the initial amplitude of the instability wave $\tilde{A}_{0}$ in equation (17), increases with jet temperature. This agrees with the calculated results of Tam and Chen [1993]. The figure indicates that jet spreading rate is changing with the operating conditions; but, that far field radiated noise at this Strouhal number is increasing with jet temperature. The results show that to increase spreading rate by changing the operating conditions does not necessarily lead to a decrease in radiated noise. As the jet temperature increases, the convected Mach number increases resulting in a shift of the measured noise radiation peak to larger angles. This trend is followed in the calculated

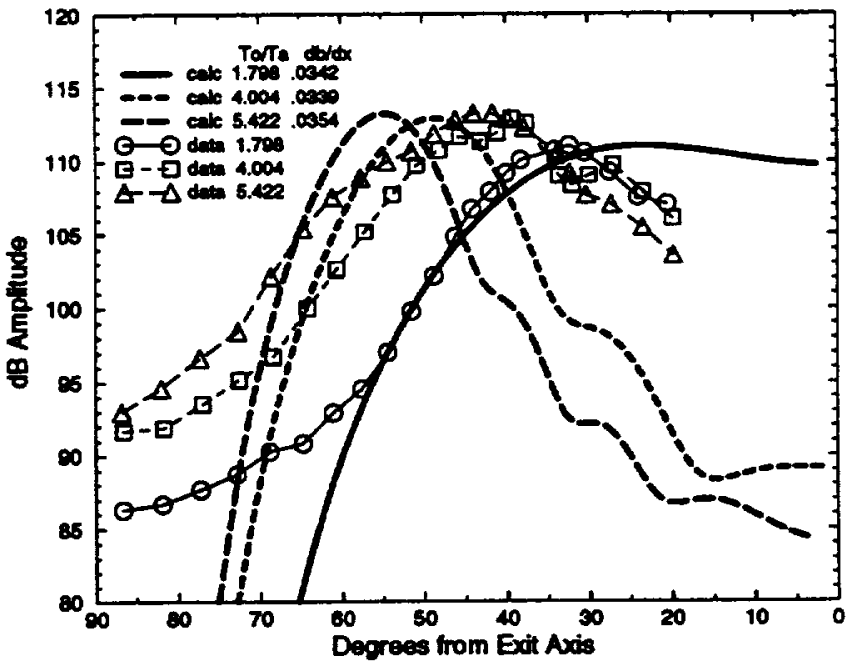

Figure 1: Comparison of $M_{j}=2$ jet calculations to measured far field noise data. $n=1, S t=.11$

results.

\subsection{Effects of Enhanced Mixing}

One method to enhance mixing is to place tabs around the nozzle lip. Kobayashi et al. [1993] ran a series of tests using various size tabs. Though their jets are underexpanded, the noise in the downstream direction is dominated by mixing noise and is affected by changes in jet spreading. In the experiment, two small tabs with $0.5 \%$ blockage are placed at the nozzle lip. Even though the tabs are small and they eliminate jet screech, the two tab arrangement could still distort the jet flow axisymmetry (See Samimy et al. [1993]). Hence, we use the measured noise data as a reference in our enhanced mixing simulation. The measured OASPL directivity patterns for a jet with and without tabs are shown in Figure 2.

To simulate enhanced mixing, $\kappa$ in equation (3) is increased above 1 . Assuming that the initial amplitude of the instability wave remains unchanged, the level of enhancement is increased until the enhanced jet far field noise radiation pattern approximately matches the measured data. Figure 2 shows the calculated radiation patterns for normal and enhanced spreading rate jets at Strouhal number 0.20 where the instability wave is near its largest total growth. The calculated results are normalized to the measured data at 20 degrees. Agreement was found for $\kappa=1.05$ resulting in a $10 \%$ increase in initial jet spreading rate $d b / d x$ where $b$ is the jet shear layer half-width. The measured data show a reduction in peak level of $4 \mathrm{~dB}$ and the calculated data show a reduction in peak level of $3 \mathrm{~dB}$ at Strouhal number 0.20 . In both cases, the peak direction remained unchanged. The enhanced spreading rate of the jet decreases the maximum amplitude of the growing instability wave re- 


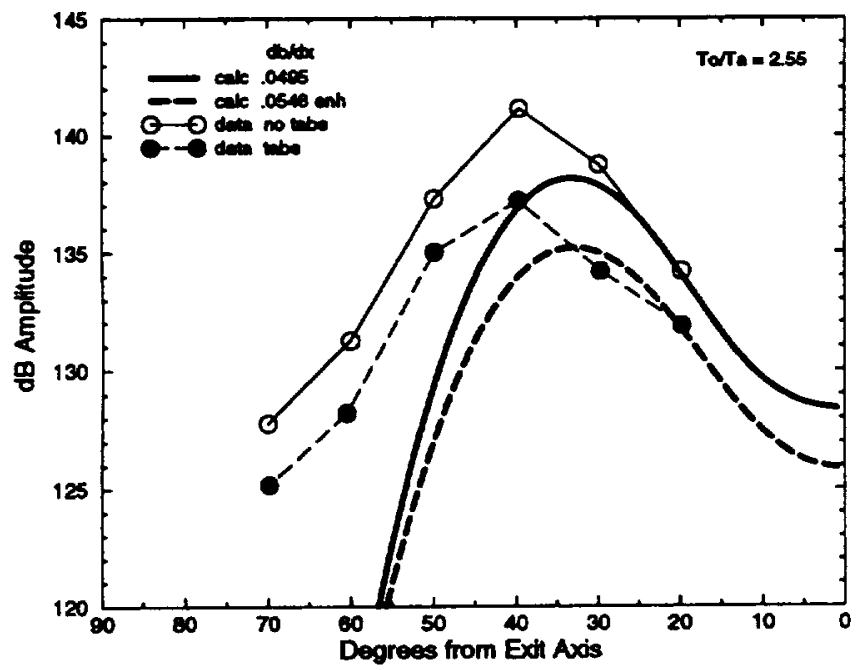

Figure 2: Effects of enhanced mixing on radiated noise, $M_{j}=1.484$. Data: OASPL, Calc.: $n=1, S t=.20$

sulting in lower levels of radiated noise.

Another means of enhancing jet mixing is to use nozzle shaping such as the elliptic nozzle referred to earlier. Noise radiation predictions for elliptic nozzles are beyond the scope of this paper, but we use measurements of far field noise radiated from elliptic jets as a basis for determining how much enhanced mixing an axisymmetric jet requires to achieve similar noise levels. Again, the measured data is in terms of OASPL. We make the calculations for a Strouhal number of 0.40. Figure 3 shows the measured far field noise radiation pattern for the reference axisymmetric jet and the elliptic jet on both the major and minor axes. The calculated data are referenced to the peak level of the measured axisymmetric jet data. The enhanced jet with $\kappa=1.05$ has reduced noise levels comparable to the elliptic jet along the minor axis, about $3 \mathrm{~dB}$. This is the same level of enhanced spreading and noise reduction achieved with tabs in Figure 2. To achieve comparable levels along the major axis, the axisymmetric jet must be enhanced with about $\kappa=1.10$ or about a $21 \%$ increase in the initial jet spreading rate. The peak noise reduction is about $5.5 \mathrm{~dB}$.

We next compare the effects of changes in spreading due to changes in operating conditions versus enhanced spreading. Figure 4 shows the measured and calculated data for the two hottest jets shown in Figure 1, ie. $T_{o} / T_{a}=4.004$ and $T_{o} / T_{a}=5.422$. Even though the initial spreading rate has increased from the cooler jet to the hotter jet, the effects of increased temperature, increased velocity, and increased convected Mach number have been to change directivity and slightly increase the radiated noise levels. If instead of changing operating conditions, the spreading rate of the cooler jet is enhanced to the same spreading rate as the hotter jet, the radiated noise levels are slightly reduced and the

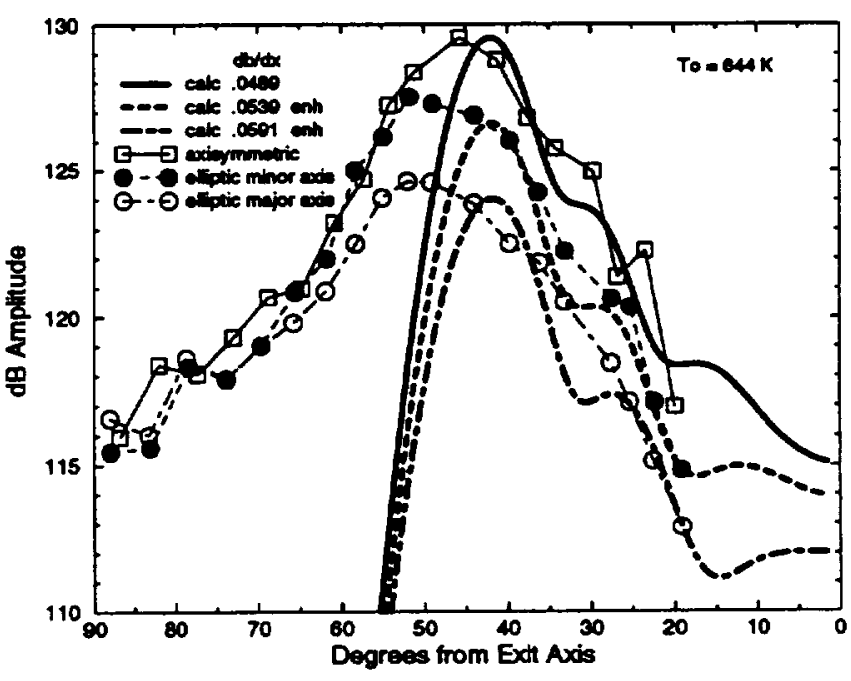

Figure 3: Effects of enhanced mixing on radiated noise, $M_{j}=1.5$. Data: OASPL, Calc.: $n=1, S t=.40$

directivity unchanged. This result again assumes that the initial instability wave amplitude remains unchanged during enhancement.

Finally, we consider the effects of simulated enhanced mixing as a function of spreading rate and Strouhal number for a hot, $M_{j}=2$ jet. Figure 5 shows changes in peak far field radiated noise levels for enhanced mixing relative to the normal spreading rate jet. Enhancing the jet spreading rate is more effective at lowering radiated noise at lower Strouhal numbers than higher Strouhal numbers. Also, the relative increases in spreading rate are less effective at noise reduction as the rate increases.

At present, it is difficult to enhance jet mixing without significantly altering the flow making the present analysis technique difficult to apply. The 10 to $20 \%$ increases in jet spreading rate we simulated are assumed to take place with minimal impact on mean flow profiles. Thus, our analysis could proceed showing calculated results that are comparable to reference measured data. For larger amounts of induced mixing and larger jet spreading rates, Kobayashi et al. [1993] showed no increases in noise reduction. Further studies are necessary to determine the limits of noise reduction achievable through enhanced mixing.

\section{SUMMARY}

In this paper, we have described a procedure for the prediction of noise from the instability waves of supersonic, axisymmetric jets. A numerical method was used to generate the developing mean flow. This provided an efficient means for obtaining the developing mean flow for various operating conditions and to simulate enhanced jet mixing. Enhancing the jet spreading rate at constant operating conditions decreased the maximum 


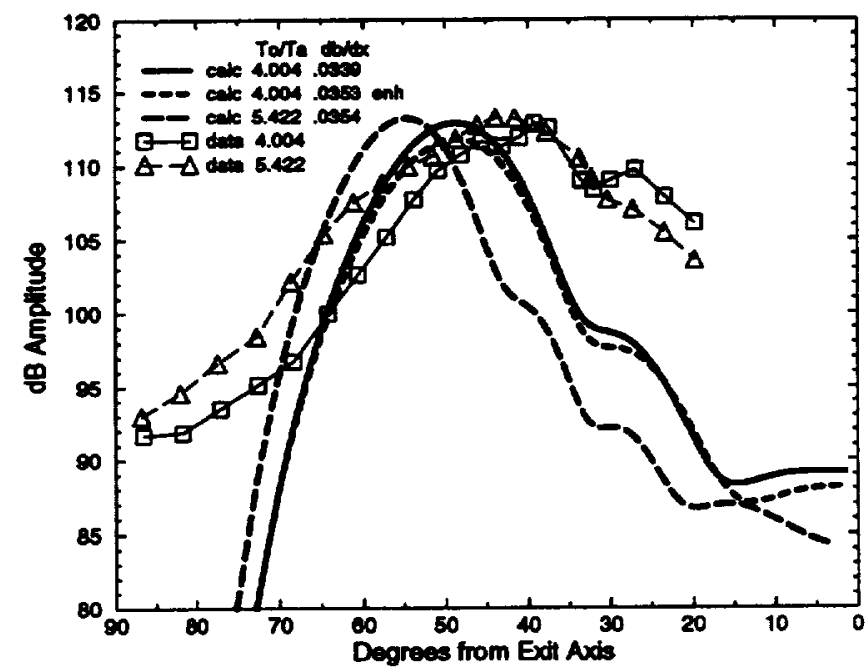

Figure 4: Effects of operating conditions versus enhanced mixing on radiated noise, $M_{j}=2 . \quad n=1$, $S t=.11$

amplitude of the growing instability wave resulting in decreased far field radiated noise. A change in operating conditions to increase the spreading resulted in noise increases compared to noise decreases for enhanced mixing to the same spreading rate.

\section{REFERENCES}

K. K. Ahuja and W. H. Brown. Shear Flow Control by Mechanical Tabs. Paper No. 89-0994, AIAA, 1989.

K. K. Ahuja. Mixing Enhancement and Jet Noise Reduction Through Tabs Plus Ejectors. Paper No. 93-4347, AIAA, 1993.

M. E. Crawford and W. M. Kays. STAN5 - A Program for Numerical Computation of Two-Dimensional Internal and External Boundary Layer Flows. CR-2742, NASA, 1976.

M. D. Dahl. The Aeroacoustics of Supersonic Coaxial Jets. PhD thesis, Penn State University, 1994.

E. Gutmark, K. C. Schadow, and K. J. Wilson. Noncircular Jet Dynamics in Supersonic Combustion. $J$. Propulsion, 5:529-533, 1989.

H. Kobayashi, H. Oinuma, T. Sawamura, and E. Outa. Effects of Tab Size on Supersonic Underexpanded Cold and Heated Jet Noise Suppression and Jet Thrust Loss. Paper No. 93-4348, AIAA, 1993.

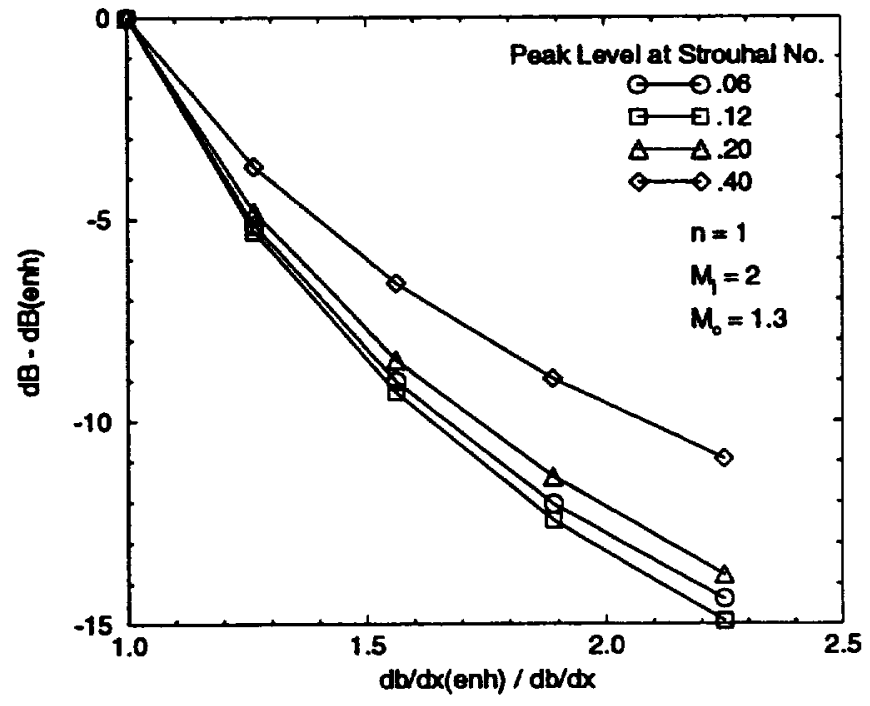

Figure 5: Example noise reductions versus enhanced spreading rate.

J. Lepicovsky, K. K. Ahuja, W. H. Brown, and R. H. Burrin. Coherent Large-Scale Structures in High Reynolds Number Supersonic Jets. CR-3952, NASA, 1985.

M. Samimy, K. B. M. Q. Zaman, and M. F. Reeder. Effect of Tabs on the Flow and Noise Field of an Axisymmetric Jet. AIAA J., 31:609-619, 1993.

J. M. Seiner and E. A. Krejsa. Supersonic Jet Noise and the High Speed Civil Tansport. Paper No. 89-2358, AIAA, 1989.

J. M. Seiner and M. K. Ponton. Aeroacoustic Data for High Reynolds Number Supersonic Axisymmetric Jets. TM-86296, NASA, 1985.

J. M. Seiner and M. K. Ponton. Supersonic Acoustic Source Mechanisms for Free Jets of Various Geometries. In Combat Aircraft Noise, AGARD-CP-512, 1991.

J. M. Seiner, M. K. Ponton, B. J. Jansen, and N. T. Lagen. The Effects of Temperature on Supersonic Jet Noise Emission. Paper No. 92-02-046, DGLR/AIAA, 1992.

J. M. Seiner, T. R. S. Bhat, and M. K. Ponton. Mach Wave Emission from a High Temperature Supersonic Jet. Paper No. 93-0734, AIAA, 1993.

C. K. W. Tam and D. E. Burton. Sound Generated by Instability Waves of Supersonic Flows. Part 2. Axisymmetric Jets. J. Fluid Mech., 138:273-295, 1984.

C. K. W. Tam and P. Chen. Turbulent Mixing Noise from Supersonic Jets. Paper No. 93-4408, AIAA, 1993. 

Public reporting bunden for this collection of information is estimated 10 average 1 hour per response, including the time lor reviewing instructions, searching existing dala sources, gathering and malntaining the data needed, and completing and reviewing the collection of information. Send comments regarding this burden estimate or any other aspect of this galection of information, including sugnestions for reducing this burden, to Washington Headquarters Services, Directorate for Information Operations and Reports. 1215 Jeflerson Davis Highway, Suhe 1204. Arlington, VA 22202-4302, and to the Office of Management and Budget, Papermork Reduction Project (0704-0188), Washington, DC 20503.

\begin{tabular}{|l|l|l|l|}
\hline 1. AGENCY USE ONLY (Leave blank) & $\begin{array}{c}\text { 2. REPOAT DATE } \\
\text { March } 1995\end{array}$ & $\begin{array}{r}\text { 3. REPORT TYPE AND DATES COVERED } \\
\text { Technical Memorandum }\end{array}$ \\
\hline
\end{tabular}

4. TITLE AND SUBTITLE

Supersonic Jet Noise Reductions Predicted With Increased Jet Spreading Rate

6. AUTHOR(S)

Milo D. Dahl and Philip J. Morris

\section{PERFORMING ORGANIZATION NAME(S) AND ADDRESS(ES)}

National Aeronautics and Space Administration

Lewis Research Center

Cleveland, Ohio 44135-3191

9. SPONSORING/MONITORING AGENCY NAME(S) AND ADDRESS(ES)

National Aeronautics and Space Administration

Washington, D.C. 20546-0001
WU-505-62-52

8. PERforming organization REPORT NUMBER

E-9491

10. SPONSORINGMONITORING AGENCY REPORT NUMBER

NASA TM-106872

11. SUPPLEMENTARY NOTES

Prepared for the Joint Fluids Engineering Conference cosponsored by the American Society of Mechanical Engineers and the Japan Society of Mechanical Engineers, Hilton Head, South Carolina, August 13-18, 1995. Milo D. Dahl, NASA Lewis Research Center, and Philip J. Morris, Pennsylvania State University, University Park, Pennsylvania 16802. Responsible person, Milo D. Dahl, organization code 2660, (216) 433-3578.

12a. DISTRIBUTIONAVAILABILTTY STATEMENT

12b. DISTRIBUTION CODE

Unclassified - Unlimited

Subject Category 71

This publication is available from the NASA Center for Aerospace Information, (301) 621-0390.

13. ABSTRACT (Maximum 200 words)

In this paper, predictions are made of noise radiation from single, supersonic, axisymmetric jets. We examine the effects of changes in operating conditions and the effects of simulated enhanced mixing that would increase the spreading rate of the jet shear layer on radiated noise levels. The radiated noise in the downstream direction is dominated by mixing noise and it is well described by the instability wave noise radiation analysis. A numerical prediction scheme is used for the mean flow providing an efficient method to obtain the mean flow development for various operating conditions and to simulate the enhanced mixing. Using far field radiated noise measurements as a reference, the calculations predict that enhanced jet spreading results in a reduction of radiated noise.

\section{SUBJECT TERMS}

Supersonic jets; Noise prediction; Noise reduction; Instability waves; Enhanced mixing

17. SECUPITY CLASSIFICATION OF REPORT

Unclassified

18. SECURTY CLASSIFICATION
OF THIS PAGE
Unclassified

19. SECURITY CLASSIFICATION OF ABSTRACT Unclassified
15. NUMBER OF PAGES 8

16. PRICE CODE

$\mathrm{AO} 2$

20. LIMATATION OF ABSTRACT

Standard Form 298 (Rev. 2-89) Prescribed by ANSI Std. Z39-18 298-102 